\title{
Consequences of blunting the mevalonate pathway in cancer identified by a pluri- omics approach
}

Sophie Goulitquer', Mikaël Croyall, Julie Lalande 3 , Anne-Lise Royer ${ }^{4}$, Yann Guitton $\mathbb{E}^{4}$, Danielle Arzur ${ }^{1}$, Stéphanie Durand ${ }^{1}$, Catherine Le Jossic-Corcos ${ }^{1}$, Alain Bouchereau ${ }^{5}$, Philippe Potin ${ }^{6}$, Serge Akoka ${ }^{3}$, Jean-Philippe Antignac ${ }^{4}$, Michel Krempf ${ }^{2}$, Véronique Ferchaud-Roucher ${ }^{2}$, Patrick Giraudeau ${ }^{3,7}$ and Laurent Corcos $^{1}$

\begin{abstract}
We have previously shown that the combination of statins and taxanes was a powerful trigger of HGT-1 human gastric cancer cells' apoptosis". Importantly, several genes involved in the "Central carbon metabolism pathway in cancer", as reported in the Kyoto Encyclopedia of Genes and Genomes, were either up- (ACLY, ERBB2, GCK, MYC, PGM, PKFB2, SLC1A5, SLC7A5, SLC16A3,) or down- (IDH, MDH1, OGDH, P53, PDK) regulated in response to the drug association. In the present study, we conducted non-targeted metabolomics and lipidomics analyses by complementary methods and cross-platform initiatives, namely mass spectrometry (GC-MS, LC-MS) and nuclear magnetic resonance (NMR), to analyze the changes resulting from these treatments. We identified several altered biochemical pathways involved in the anabolism and disposition of amino acids, sugars, and lipids. Using the Cytoscape environment with, as an input, the identified biochemical marker changes, we distinguished the functional links between pathways. Finally, looking at the overlap between metabolomics/lipidomics and transcriptome changes, we identified correlations between gene expression modifications and changes in metabolites/lipids. Among the metabolites commonly detected by all types of platforms, glutamine was the most induced (6-7-fold), pointing to an important metabolic adaptation of cancer cells. Taken together, our results demonstrated that combining robust biochemical and molecular approaches was efficient to identify both altered metabolic pathways and overlapping gene expression alterations in human gastric cancer cells engaging into apoptosis following blunting the cholesterol synthesis pathway.
\end{abstract}

\section{Introduction}

Cancer cell metabolism shows strong alterations required for cell activity and growth ${ }^{2}$, including a high avidity for glucose and lipids ${ }^{3-5}$. Statins, cholesterollowering drugs used to prevent cardiovascular diseases, are competitive inhibitors of 3-hydroxy-3-methyl-glutaryl-coenzyme A reductase (HMG-CoA Red). They block mevalonate production, in a rate-limiting step of the

Correspondence: Laurent Corcos (laurent.corcos@inserm.fr)

'Génétique, Génomique Fonctionnelle et Biotechnologies, INSERM, Université de Brest, EFS, Brest, France

${ }^{2}$ INRA UMR 1280, Centre de Recherche en Nutrition Humaine de I'Ouest

(CRNHO), CHU Hôtel-Dieu, Université de Nantes, Nantes, France

Full list of author information is available at the end of the article.

Edited by A. Stephanou cholesterol synthesis pathway ${ }^{6}$. In addition, statins induce apoptosis of many cancer cell types, an effect counteracting the addiction of cancer cells to pathways driving cell division, motility, and proliferation ${ }^{7}$. Indeed, inhibition of HMG-CoA Red results in a shortage of several important metabolites, including Farnesyl PyroPhosphate (FPP) and GeranylGeranyl PyroPhosphate (GGPP) ${ }^{7,8}$. FPP and GGPP bind, in a post-translational manner (prenylation), to the $\mathrm{C}$-terminus of a restricted set of proteins from the Ras and Rho families, which makes those proteins migrate and anchor to the plasma membrane, where they acquire GTPase activity. Prenylation blockade results in restriction of the positive growth signals associated with the MAP kinase-dependent pathways ${ }^{9}$. Docetaxel, an

\section{(c) The Author(s) 2018}

(c) (i) Open Access This article is licensed under a Creative Commons Attribution 4.0 International License, which permits use, sharing, adaptation, distribution and reproduction cc) in any medium or format, as long as you give appropriate credit to the original author(s) and the source, provide a link to the Creative Commons license, and indicate if changes were made. The images or other third party material in this article are included in the article's Creative Commons license, unless indicated otherwise in a credit line to the material. If material is not included in the article's Creative Commons license and your intended use is not permitted by statutory regulation or exceeds the permitted use, you will need to obtain permission directly from the copyright holder. To view a copy of this license, visit http://creativecommons.org/licenses/by/4.0/. 
anticancer taxane compound used to treat breast and gastric cancer ${ }^{10}$, promotes microtubules assembly and stabilizes the polymers against depolymerization, thereby inhibiting microtubule dynamics ${ }^{11}$.

We reported that both lovastatin and docetaxel triggered efficient apoptosis of HGT-1 human gastric cancer cells, but also colon or liver cancer cells ${ }^{1}$. The combination of lovastatin and docetaxel resulted in a synergistic apoptotic effect, as compared to either compound alone. Furthermore, supplementing adenocarcinoma cells with FFP or GGPP prevented statin-dependent apoptosis of several cancer cell types, including gastric cancer cells ${ }^{12}$. Finally, we showed that lovastatin triggered numerous gene expression changes, whereas docetaxel had little effects ${ }^{1}$.

Based on these results, we decided to investigate the potential of these drugs to alter the metabolomics and lipidomics of early cancer cell engagement into apoptosis. Such markers would hold promise as a characteristic signature of changes resulting from treatment with these drugs and, potentially, with other cytotoxic drugs. To this end, we embarked on a collaborative project with mass spectrometry (GC-MS and LC-MS) and Nuclear Magnetic Resonance (NMR) platforms from the CORSAIRE metabolomics and lipidomics network (https://www. biogenouest.org/en), in a non-targeted approach, to determine the modifications of metabolomics and lipidomics profiles in HGT-1 cells. The levels of more than 100 metabolites and lipids were significantly altered by the treatments, including amino acids, organic acids, sugars, and several families of lipids. Furthermore, we identified a small set of compounds with altered accumulation levels jointly recognized by all analytical platforms, thereby conferring robustness to our methodological framework. Strikingly, most changes were due to lovastatin treatment, used alone or with docetaxel, which had little effect by itself. Finally, we combined metabolomics/lipidomics and transcriptomics data obtained from lovastatin and/or docetaxel-treated cells, which pointed to key biochemical alterations and to the recognition of novel biomarkers of the onset of cancer cell apoptosis.

\section{Results}

All experiments were performed with extracts from cells treated by the drugs for $36 \mathrm{~h}$, with the exception of the kinetics experiments $(17,24$, and $36 \mathrm{~h})$.

\section{HMG-CoA reductase enzyme activity following drug treatments}

In order to evaluate the effects of lovastatin and/or docetaxel on HMG-CoA Red activity, we determined the level of mevalonate produced. As expected, any condition that included lovastatin resulted in full absence of HMGCoA Red activity. The basal enzyme activity was $18 \pm 2$ $\mathrm{pmol} / \mathrm{min} / \mathrm{mg}$ protein. Docetaxel had no effect on this basal activity, and did not overcome the suppression resulting from lovastatin.

\section{Cross-platform principal component analysis}

In order to see if the treatment of HGT-1 cells by lovastatin and/or docetaxel modified the metabolome and lipidome, we performed Principal Component Analyses (PCA) using, as a first entry, harmonized metabolomics data following Pareto and $\log 10$ transformation to have data from each platform within a similar order of magnitude ${ }^{13}$. No marker selection was performed upstream of the PCA analysis. In addition, a PCA analysis was conducted before and after normalization, which showed that the normalization step did not modify the overall discriminatory effects of the treatments. A strong separation was observed between conditions with lovastatin and without lovastatin in HGT-1 (Fig. 1) but also in AGS human gastric cancer and HCT116 human colon carcinoma cells (Supplementary Figure 1).

Because of their distinct modes of action, lovastatin, and docetaxel were not necessarily expected to both trigger large and convergent metabolomics/lipidomics changes. Whereas docetaxel acts mechanically onto microtubule dynamics and induced only a few gene expression changes, lovastatin strongly altered several gene expression programs ${ }^{1}$. Although the metabolome/lipidome changes may not necessarily converge onto common gene expression-driven alterations, this observation could explain our results, at least in part.

\section{Comparison of metabolomics data among analytical platforms}

In order to compare the results from all platforms, we developed a specific strategy to standardize the data. First, the LC-MS (Fig. 1A, B), GC-MS (Fig. 1C), and NMR (Fig. 1D) raw data were collected and standardized by the amounts of proteins. The analytical drift was then corrected using Workflow4Metabolomics, operating under the Galaxy environment, by a Van Der Kloet transformation ${ }^{14}$. As each data table contained intensities expressed as arbitrary units specific for each instrument, we used a $\log 10$ and then a Pareto standardization for data acquired by mass spectrometry and only Pareto for NMR data. The NMR results were somewhat distinct from those of the MS platforms, but the PCA analysis showed that NMR data were consistent with the (control, docetaxel) vs. (lovastatin, lovastatin + docetaxel) discrimination also observed by the MS platforms (Fig. 1d). Among the NMR signals, some were identified while others, identified by both GC-MS and LC-MS, remained unseen by NMR (Supplementary Table 1). That NMR- and MS-based metabolomics results were not entirely overlapping could result from the different 


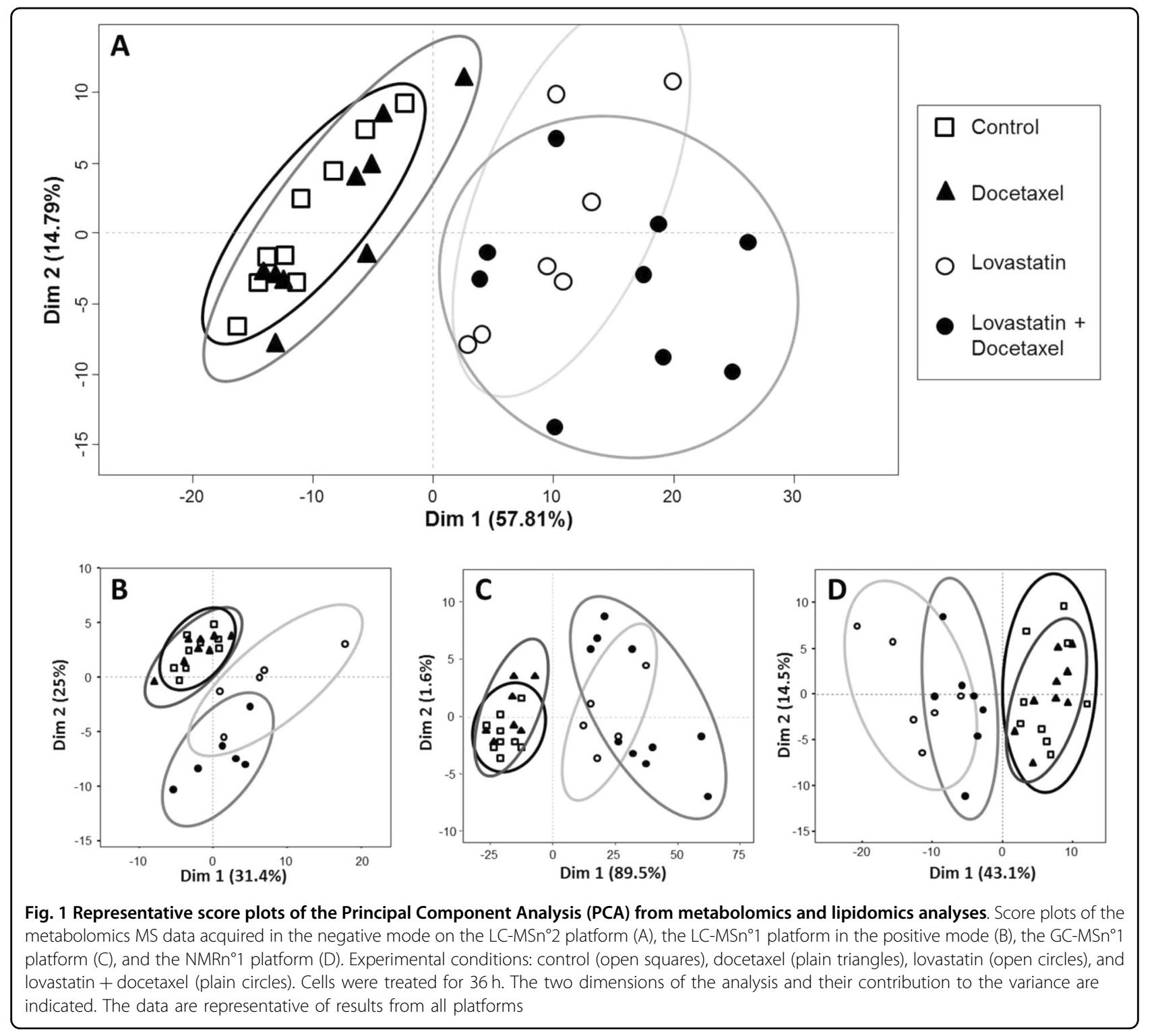

physico-chemical properties of the compounds, or be linked to the fact that MS often detects minor species inaccessible to NMR. The data matrices that correlated most with each other were those from the LCMSn 2 platform: $\mathrm{RV}_{\text {(LC-MSn }} 2$ LLipidoPos_vs_LC-MSn'2LipidoNeg) $=0.978$ and $\mathrm{RV}_{\left(\mathrm{LC}-\mathrm{MSn}{ }^{\circ} 2 \mathrm{LipidoPos} \text { vs }{ }_{\mathrm{LC}-\mathrm{MSn}}{ }^{\circ} \mathrm{Neg}\right)}=0.886$, $\mathrm{RV}_{\text {(LC-MSn } 2 \text { 2LipidoNeg_vs_LC-MSn'2Neg) }}=0.870$ (Fig. 2).

Metabolomics data generated by the same platform but in different ionization modes (positive or negative) showed partial correlation, such that the intra-platform $\mathrm{RV}$ was 0.257 on the $\mathrm{LC}-\mathrm{MSn}^{\circ} 1$ platform, and 0.342 on the $\mathrm{LC}-\mathrm{MSn}^{\circ} 2$ platform, even though the data were obtained from the same samples with a single extraction process. The data matrix size could explain the low correlation scores. Indeed, on the $\mathrm{LC}-\mathrm{MSn}^{\circ} 1$ platform, 1598 signals were extracted from the raw data in the positive mode, but only 613 signals in the negative mode. These low correlations scores likely reflected the fact that metabolomics extractions were more efficient for polar metabolites, which were separated by acidified phases in liquid chromatography, promoting a better ionization in the positive ion mode, including adducts, and thus, producing more detectable ions.

\section{Integration of metabolomics and lipidomics data}

To address the consequences of the variations detected by the platforms, we performed PCAs for each platform, focusing on the coordinates from the first component of the PCAs, which explained most of the variance. We used these PCAs as information compression to reduce a matrix of data to a vector (defined by the coordinates of the sample in the first component) (Supplementary 


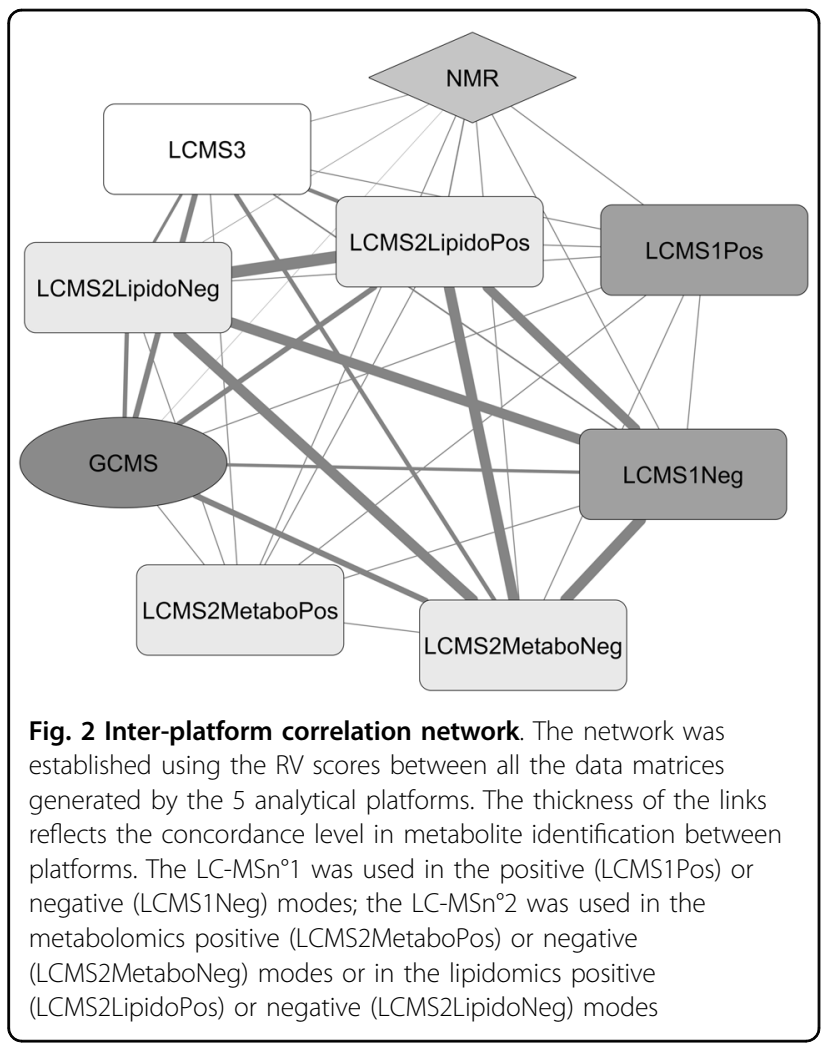

Figure 2). Using the first two components, the PCA explained $70 \%$ of the variance and, importantly, discriminated two large data sets (control, docetaxel) vs. (lovastatin, lovastatin + docetaxel) (Fig. 3). Hence, the global information obtained by each platform was convergent, and each platform would be able to determine whether a sample was treated at least by lovastatin. However, it was almost impossible to distinguish a control from a docetaxel-treated sample, or a lovastatin from a (lovastatin + docetaxel)-treated sample. However, our metabolomics approach also identified discrete and discriminant biomarkers.

\section{Metabolites and lipids identification}

To determine the nature of the metabolites and lipids specifically altered by each treatment condition, several databases were queried, including the local database (NIST), online databases (LipidMaps, MassBank, HMDB) or in-house databases ${ }^{15}$. One hundred and eleven distinct compounds-some being detected by more than one platform, including amino acids, small organic acids, sugars, spermidine, phosphatidyl-choline or triglycerides-were sorted out across the nine data matrices generated by all platforms (Table 1). Depending on the metabolite considered, changes in its intracellular content varied specifically with the treatments. For instance, glutamine was increased in lovastatin or

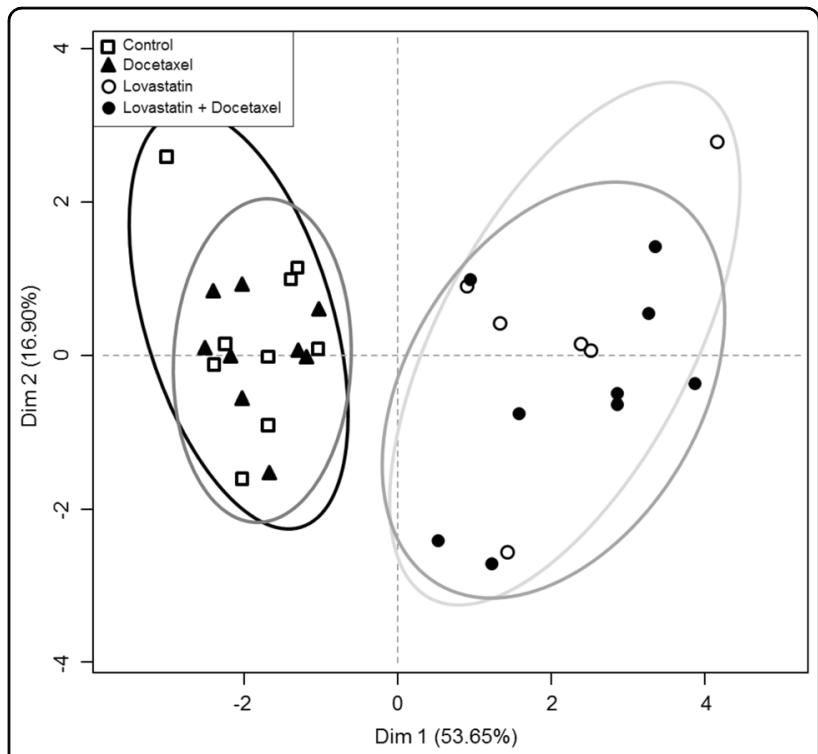

Fig. 3 Score plot of the "meta" PCA prepared from the calculated Dim1 coordinate from primary PCA analyses resulting from each data matrix. Experimental conditions: control (open squares), docetaxel (plain triangles), lovastatin (open circles) and (lovastatin + docetaxel) (plain circles). Cells were treated for $36 \mathrm{~h}$. The two dimensions of the analysis and their contribution to the variance are indicated

(lovastatin + docetaxel) extracts. Spermidine was decreased with (lovastatin + docetaxel). This dedicated biomarker identification allowed to better discriminate the different treatments than the global analysis of metabolomics profiles.

It was expected that levels of some lipids should be modified in response to statins. We thus analyzed intracellular lipidomes by two of the platforms (LC-MSn 2 and $\mathrm{LC}-\mathrm{MSn}^{\circ}$ 3), using the Bligh \& Dyer extraction procedure $^{16}$. Four classes of metabolites were identified as significantly separating out the (lovastatin + docetaxel) condition from the control or docetaxel groups (Fig. 4): ceramide [Cer $(\mathrm{d} 18: 1 / 16: 0)]$, phosphatidylcholines [PC (28:0), $\mathrm{PC}(30: 1), \mathrm{PC}(32: 2), \mathrm{PC}(34: 3)$ and $\mathrm{PC}(34: 5)]$, triglycerides [TG(52:2), TG(52:3) and TG(54:2)] and sphingomyelins [SM(16:0), $\operatorname{SM}(18: 0), \operatorname{SM}(20: 0), \operatorname{SM}(22: 0)$, $\operatorname{SM}(24: 0), \operatorname{SM}(18: 1), \operatorname{SM}(20: 1), \operatorname{SM}(22: 1)$, and $\operatorname{SM}(24: 1)]$. The amount of $\mathrm{PC}(30: 1)$ and $\mathrm{PC}(32: 2)$, detected in the positive mode, was reduced by (lovastatin + docetaxel). Conversely, [Cer(d18:1/16:0)] was increased in all cases. From the 19 triglycerides species detected, only 3 were modulated between treatment groups. No significant differences were observed between treatments for the sum of all detectable triglycerides, except a small decrease for docetaxel alone as compared to (lovastatin + docetaxel) (Supplementary Figure 3). 
Table 1 Evolution of the 111 metabolites identified in the (lovastatin + docetaxel) vs. control condition after $36 \mathrm{~h}$ of treatment

\begin{tabular}{|c|c|c|c|c|}
\hline \multicolumn{4}{|l|}{ Up } & \multirow{2}{*}{$\begin{array}{l}\text { Down } \\
\text { Choline }\end{array}$} \\
\hline 2,3-Dihydroxypropyl palmitate & Cis-4-hydroxy-D-proline & L-Glutamine & Phosphoric acid & \\
\hline 2-Hydroxy-3-Methylbutyric acid & Citric acid & L-Homoserine & Propionyl-L-carnitine & Fumarate \\
\hline 2-Oxovaleric acid & Creatine & L-Isoleucine & Pyridoxal & L-Glutathion \\
\hline 3-Hydroxytyramine & Creatinine & L-Leucine & Pyridoxine & L-Tryptophan \\
\hline 3-Methyl-2-oxobutanoic acid & Cyclohexene-3,5-dione & L-Lysine & Pyroglutamic acid & Pantothenic acid \\
\hline 4-Methyl-2-oxovaleric acid & Cystathionine & L-Methionine & Pyrophosphate & Spermidine \\
\hline 5-Aminovaleric acid & Dehydroabietic acid & L-Phenylalanine & Ribothymidine & Threonine \\
\hline 5-Deoxy-5-methylthioadenosine & D-Fructose & L-Proline & Sarcosine & SM 16:0 \\
\hline 5-Methylcytidine & D-Glucose & L-Tyrosine & Stearoylglycerol & SM 18:0 \\
\hline Acetate & Dihydrouracil & Maleic acid & Succinate & SM 20:0 \\
\hline Adenosine & D-Ribose & Methylmalonic acid & Talose & SM 22:0 \\
\hline Adipic acid & Formamide & Monopalmitoylglycerol & Taurine & SM 24:0 \\
\hline Alpha-hydroxyisobutyric acid & Fructose di-phosphate & Myo-inositol & TG (52:2) & SM 18:1 \\
\hline Alpha-ketoglutaric acid & Glutamate & Myristic acid & TG (52:3) & SM 20:1 \\
\hline Aminoadipic acid & Glyceraldehyde & $N$-acetyl-asp-Glu & TG (54:2) & SM 22:1 \\
\hline Aminomalonic acid & Glycerol stearate & N-acetyl-D-glucosamine & Tryptophan & SM 24:1 \\
\hline Arginine & Glycerylphosphorylcholine & $\mathrm{N}$-acetyl-L-aspartate & Tyramine & PC (28:0) \\
\hline Asparagine & Hexadecanoic acid & $\mathrm{N}$-acetyl-L-glutamate & Uric acid & PC (30:1) \\
\hline Aspartate & Hippuric acid & Nicotinamide & Uridine & PC (32:2) \\
\hline Azelaic acid & Hydroxybutyric acid & O-acetyl carnitine & Uridine-5'-monophosphate & PC (34:3) \\
\hline Beta-Alanine & Hypotaurine & Ornithine & Valine & PC (34:5) \\
\hline Betaine & Lactic acid & O-succinyl-L-homoserine & & \\
\hline Cer(d18:1/16:0) & L-Alanine & Phosphoglyceric acid & & \\
\hline
\end{tabular}

HGT-1 cancer cells were treated for $36 \mathrm{~h}$ and collected for biochemical analyses. The identified metabolites (fold-change $>2, p$-value $<0.05$ ) are listed in alphabetical order, both in the "Up" and in the "Down" columns

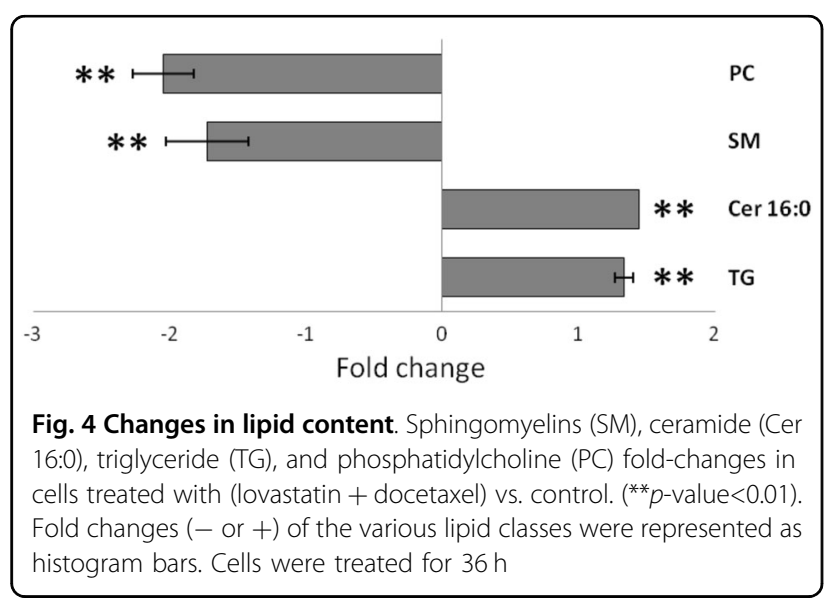

\section{Specifics of the analytical technologies}

Various compounds were identified by several platforms. Hence, in addition to the ability of the platforms to obtain a common descriptive envelope of the metabolome, we could also identify changes in the metabolomics content with the same accuracy, thereby conferring both convergence and robustness. In contrast, some compounds were detected by only one platform (e.g. acetate, choline, or formamide identified by NMR). Nevertheless, the unique ability of a given platform to identify specific metabolites added complementary information. To analyze the overlap in metabolites detected by all technologies, we established a Venn diagram (Fig. 5). Among all of the 111 identified biomarkers, six were common: glutamate, glutamine, myo-inositol, creatine, lactic acid, and fumarate (Table 2). Moreover, 12 metabolites were detected both by GC-MS and LC-MS, but not by NMR, including citric acid, pantothenic acid, glucose, L-proline, or L-methionine (Supplementary Table 1). Finally, we combined the metabolomics and lipidomics data and generated a network (Fig. 6). Although ceramides were 


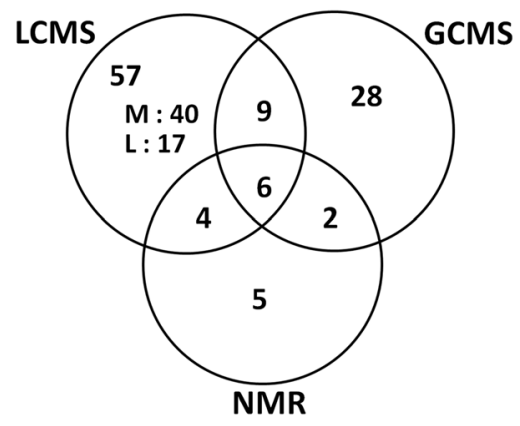

Fig. 5 Venn diagram of the specific and shared metabolites (M) and lipids (L). Metabolites detected by each technology and lipids detected by the LC-MS technology in the (lovastatin + docetaxel, 36 h) condition vs. control (fold change $>1.5, p$-value $<0.05$ ). The six metabolites common to LCMS2 and NMR were: glutamate, glutamine, myo-inositol, creatine, lactic acid, and fumarate

Table 2 Univariate quantitative analysis of metabolites commonly detected by all platforms

\begin{tabular}{|c|c|c|c|c|c|c|}
\hline & \multicolumn{2}{|l|}{ GCMS } & \multicolumn{2}{|l|}{ LCMS } & \multicolumn{2}{|l|}{ NMR } \\
\hline & $\begin{array}{l}\text { Fold } \\
\text { change }\end{array}$ & $t$-test & $\begin{array}{l}\text { Fold } \\
\text { change }\end{array}$ & $t$-test & $\begin{array}{l}\text { Fold } \\
\text { change }\end{array}$ & $t$-test \\
\hline Creatine & 2.05 & 0.0015 & 2.50 & 0.0476 & 2.17 & 0.0003 \\
\hline Fumarate & 2.56 & 0.0143 & 2.34 & 0.0000 & 1.53 & 0.0114 \\
\hline Glutamate & 1.72 & 0.0013 & 1.98 & 0.0000 & 1.53 & 0.0096 \\
\hline Glutamine & 6.52 & 0.0009 & 7.12 & 0.0000 & 6.60 & 0.0129 \\
\hline Lactic acid & 3.08 & 0.0038 & 3.96 & 0.0058 & 4.30 & 0.0275 \\
\hline Myo-inositol & 2.56 & 0.0011 & 1.61 & 0.0220 & 1.68 & 0.0034 \\
\hline
\end{tabular}

HGT-1 cancer cells were treated for $36 \mathrm{~h}$ and collected for biochemical analyses. The identified metabolites are listed in alphabetical order. The fold-changes and the $p$-values (Student $t$-test, $p<0.05$ ) are shown

modestly linked to other members of the network, a strong density of partner compounds was observed, stressing the close interplay between these lipid classes and the other biochemical categories of metabolites.

\section{Kinetic effects}

We used quantitative GC-MS data for these experiments. Each set of data was normalized with respect to its own control at the same time of treatment. We analyzed 31 metabolites from cells treated by (lovastatin + docetaxel) for all time-points. The (lovastatin + docetaxel) treatments were best separated from their respective $17 \mathrm{~h}$ and $24 \mathrm{~h}$ controls according to the first dimension of the PCA (Fig. 7), like for cells treated by lovastatin alone at the same time points (data not shown). The first dimension of the PCA, which explained the largest fraction of the variance, showed a clear separation of the (lovastatin + docetaxel) condition at $36 \mathrm{~h}$, from either the control or the drug combination at earlier time-points. Strikingly, nearly all metabolites were increased for the $36 \mathrm{~h}$ timepoint, suggesting increased synthesis over time (Supplementary Table 2). By contrast, fumarate and pantothenic acid levels were decreased at all time-points.

\section{Overlap between transcriptome changes and metabolites/ lipids altered levels}

Metabolites with a fold change $>1.5$ and a $p$-value $<0.05$ (lovastatin + docetaxel condition vs. control) and genes with a fold change $>2$ (lovastatin + docetaxel condition vs. control) were integrated into a global network using the Stitch software ${ }^{17}$ for interaction analysis of metabolites and proteins, and the Cytoscape environment for visualization ${ }^{18}$. This highlighted links between "cholesterol homeostasis" and "cellular carbohydrate biosynthetic processes" through intermediates from the "response to transition metal particles" and "female gonad development". "Cellular amino acids metabolic processes" appeared unlinked to these pathways (Supplementary Figure 4).

We next used the $\mathrm{ClueGO}^{19}$ application that extracts the non-redundant biological information for large clusters of genes, and PaintOmics ${ }^{20}$, which permits overlay transcriptomics and metabolomics data onto KEGG pathways. We identified several significantly affected pathways (Supplementary Information and Supplementary Figures 5-10). While most metabolites were increased, fumarate was decreased. Interestingly, expression of several genes from these pathways was either increased or decreased by the treatment (Supplementary Figures 5, 7-10), whereas one pathway showed no modifications in gene expression, while displaying metabolite changes (Supplementary Figure 6).

Finally, integrating our data into the general pathway of "Central carbon metabolism in cancer" (http://www. genome.jp/kegg-bin/show_pathway?hsa05230) highlighted several genes that were up-regulated by the (lovastatin + docetaxel) treatment, including the $A C L Y$, ERBB2, GCK, MYC, PKFB2, SLC1A5, SLC7A5, and the $S L C 16 A 3$ genes, together with several modified metabolites identified by one or more platforms, such as amino acids, citrate, lactate, fumarate, 2-oxoglutarate or succinate. Importantly, several other genes were down-regulated, like P53, IDH, OGDH, $M D H 1$, and $P D K$. From these observations, it can be anticipated that glucose uptake, amino acid synthesis and lactate production be increased, together with a drop in the P53-dependent sensitization to apoptosis and a stimulation of cell division through activation of the ERBB2-dependent MAP kinase pathway (Fig. 8).

\section{Discussion}

The identification of biomarkers of response to anticancer treatments is particularly important to predict 


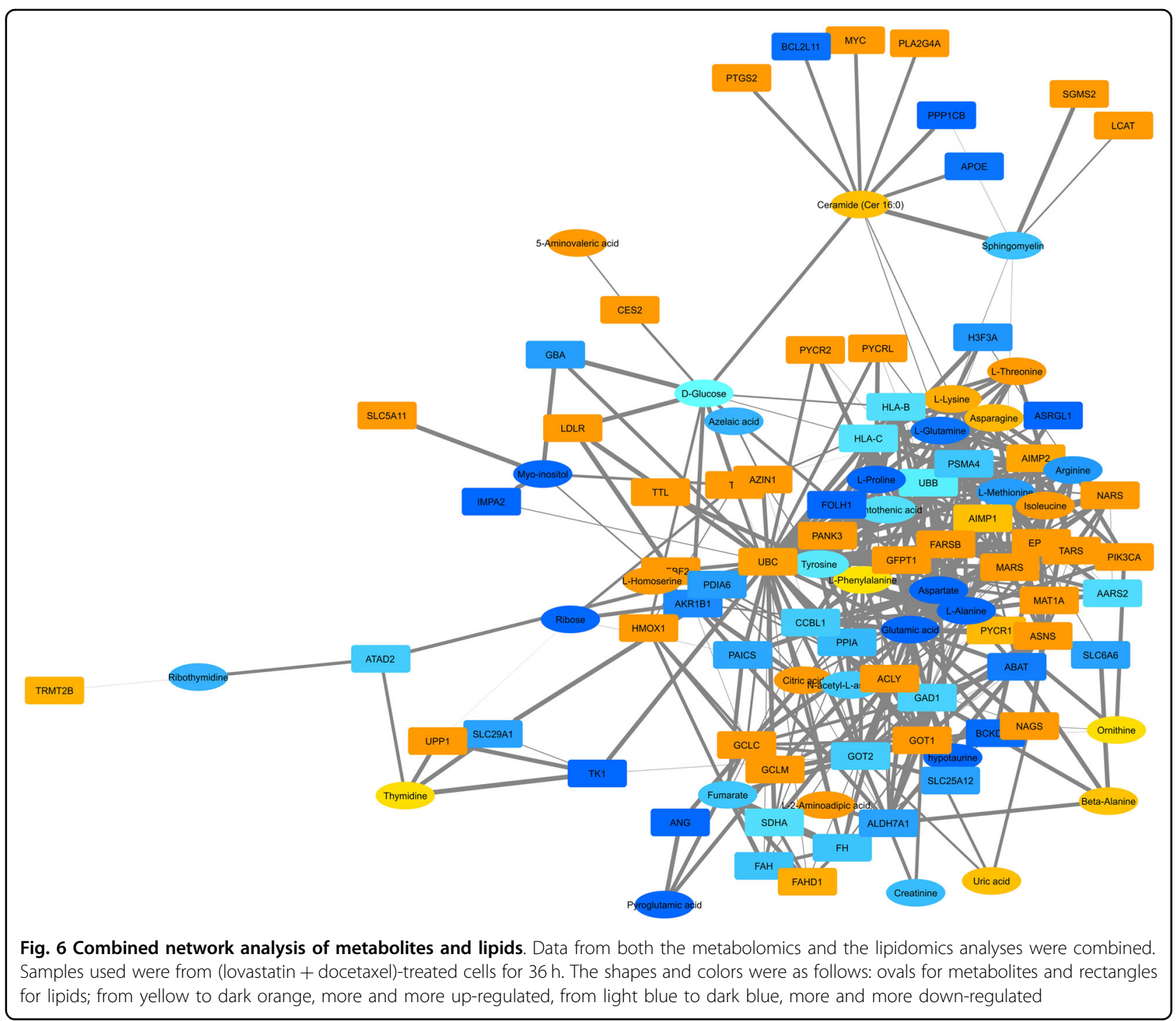

patient outcome. Model cancer cell systems are one such tool to decipher the effects of treatments onto cellular metabolic activity. In a previous study, we identified the (lovastatin + docetaxel) combination as a novel means to trigger human HGT-1 gastric cancer cells apoptosis quite efficiently ${ }^{1}$. Here, we set out to establish a robust description of metabolomics/lipidomics alterations in response to the drugs, as an initial step towards further biomarker characterization. As such, identified metabolites (Table 2) will be further explored as coherent biomarkers of drug response.

To address the possibility that the metabolome and the lipidome of gastric cancer cells in vitro could show specific alterations in response to lovastatin and/or docetaxel, we selected independent MS and NMR platforms. There was a good concordance between the results from these platforms that identified both a comparable trend of variations, and several of the same metabolites, as especially shown by the clear distinction between treatments containing or not lovastatin. Importantly, a similar discrimination between the control and the (lovastatin + docetaxel) conditions was also observed here in two other cancer cell lines from the stomach and the colon (Supplementary Figure 1). Almost all metabolite levels decreased during the first $24 \mathrm{~h}$, but rose afterwards, at 36 $\mathrm{h}$ of treatment, possibly indicating a synthesis rebound phase (Supplementary Table 2). However, both fumarate and pantothenic acid levels were decreased at all time points.

The metabolism of lovastatin has been amply described $^{21-23}$. Docetaxel is extensively metabolized by hepatic cytochromes P-450 (CYP), particularly CYP3A and CYP2C ${ }^{24}$ in human liver. The identified metabolites and lipids whose levels were modified in response to the drugs were distinct from the metabolic products of lovastatin and docetaxel. Several metabolites from the tricarboxylic 


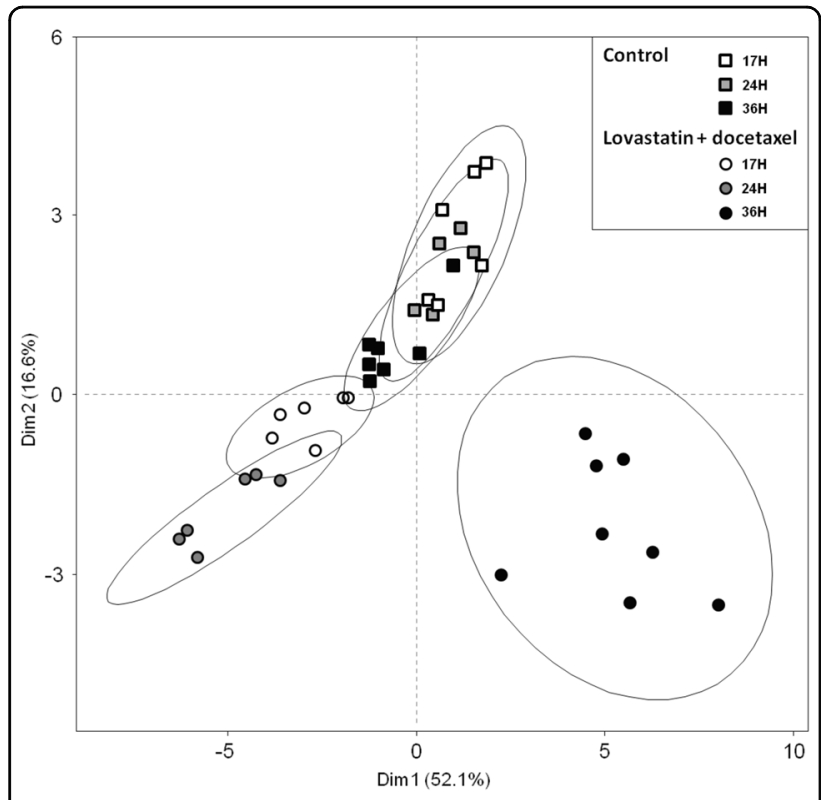

Fig. 7 PCA analysis of the kinetic effects of the (lovastatin + docetaxel) combination. The cells were treated with both drugs for the indicated times $(\mathrm{H})$ and cell extracts were prepared for GC-MS analysis. Seventeen-hours control (open squares), $24 \mathrm{~h}$ control (gray squares), $36 \mathrm{~h}$ control (black squares), $17 \mathrm{~h}$ treament (empty circles), $24 \mathrm{~h}$ treatment (gray circles), and $36 \mathrm{~h}$ treatment (black circles). Three biological replicates of each treatment condition were performed acid (TCA) cycle were raised, indicating that its activity was higher than in control cancer cells, hence closer to that of normal cells. We might suggest that this "normallike" metabolic condition may be incompatible with the mutations or epigenetic changes that drove cancer cells to switch from oxidative phosphorylation to aerobic glycolysis, participating in apoptotic cell death in response to the drugs.

Several lipid classes were altered by the treatments, including phosphatidylcholines and sphingomyelins (decreased), ceramides, and triglycerides (increased). Reportedly, lovastatin decreased phosphatidylcholine concentration by inhibiting cytidylyltransferase activity In human endothelial cells ${ }^{25}$. Ceramides, which have a well-defined pro-apoptotic role, are highly produced during apoptosis, mostly through the action of sphingomyelinase, and induce sphingomyelinase, which converts sphingomyelin back into ceramides ${ }^{26,27}$, leading to amplifying apoptotic signals in cells treated by anticancer agents ${ }^{28,29}$. The rises observed here were likely attributable, at least in part, to the apoptosis-inducing ability of lovastatin, or to the statin-dependent increase in ceramide synthase ${ }^{30}$.

Docetaxel binds tubulin, thereby preventing microtubule repolymerization, provoking mitotic arrest and cell death $^{31}$. It decreased expression of several genes in breast

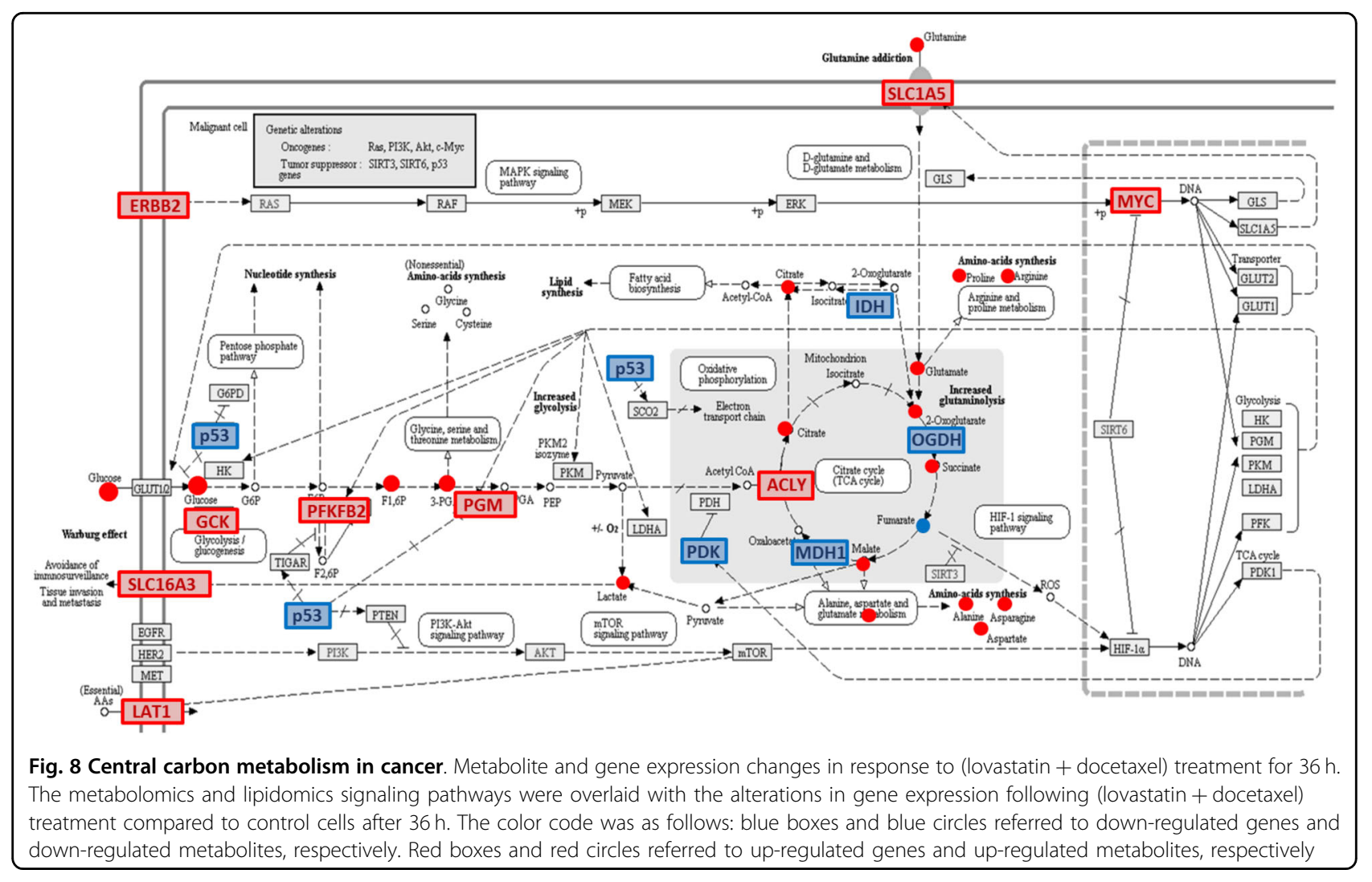


cancer cells, including the $B C L X$ and $B C L 2$ anti-apoptotic genes $^{32}$. In addition, paclitaxel, another taxane, downregulated several lipid synthesis genes in ovarian cancer, including the APOE, HMGCS1, and LDLR genes, indicating that docetaxel could also contribute to alterations of lipid levels ${ }^{33}$. In paclitaxel-treated HeLa cells, a drop in phosphatidylcholine was observed ${ }^{13}$, which also occurred here in docetaxel-treated HGT-1 cells.

Lovastatin increased metabolites from the TCA cycle in cancer cells (from the ovary), but reduced the metabolites associated with glycolysis. Nearly all, among metabolites involved in the TCA cycle, together with amino-acids, were up-regulated similarly by lovastatin in this study ${ }^{34}$ and by (lovastatin + docetaxel) in our study, including citrate, lactate, malate, succinate, tryptophan, and valine.

We showed previously that treatment with lovastatin and/or docetaxel engaged cells from several cancer types into apoptosis, in addition to gastric cancer cells, including cervix, lung, or liver cancer cells ${ }^{1}$. Because most of the metabolomics and lipidomics changes observed in response to (lovastatin + docetaxel) could not be distinguished from those resulting from lovastatin alone, we surmise that any lovastatin-responsive cancer cell type should show comparable metabolomics and lipidomics profiles.

To determine the links between both metabolomics/ lipidomics and gene expression data types, we used PaintOmics ${ }^{20}$. Several of the biochemical pathways showing variations in activity were indeed associated with selected changes in mRNA levels (see Supplementary Information and Supplementary Figures 5, 7-10). Although most modifications in biochemical pathways were associated with alterations in gene expression, other altered pathways were apparently unlinked to gene expression changes, possibly because their variation in expression remained undetectable, or because the cell response to (lovastatin + docetaxel) did not require adjustment of mRNA levels of genes from the considered pathways, e.g. for the "amino acids biosynthesis" pathway (Supplementary Figure 6).

Importantly, glucose and glutamine levels, two major sources of energy for cancer cells ${ }^{3,35}$, were increased, so was citrate-a precursor of fatty acids-and lactate-a promoter of invasive potential. Hence, the (lovastatin + docetaxel) treatment of HGT-1 cells did not oppose the normal regulation of energy supply characteristic of cancer cells. Rather, it amplified production or capture of metabolites important for cancer cell metabolism. Because these metabolites' levels dropped at the early time-points, their further rise at $36 \mathrm{~h}$ could be interpreted as a rebound phase, which was, however, insufficient to allow cell survival, but possibly pointing to the ability of these cells to try developing survival strategies.
In view of the multiplicity of alterations occurring both at the transcriptome and metabolome/lipidome levels, and not knowing if these were a driver or a consequence of apoptosis engagement, or a mixture of both, it will be difficult to obtain a fair appreciation of which of these altered pathways would be best targeting for tumor growth inhibition in vivo. Provided the observed association between metabolite and gene variation levels were linked, at least in part, it could be envisioned to modulate expression of some of the genes deregulated under our pro-apoptotic conditions to directly address their roles in cell death. Although it may seem counterintuitive that apoptosis resulting from the (lovastatin + docetaxel) treatment was associated with a drop in P53 gene expression, this might be unlinked to HGT-1 sensitivity to apoptosis since these cells carry an inactivating P53 mutation. Furthermore, we observed that P53-proficient HCT116 colon carcinoma cells were also highly sensitive to the (lovastatin + docetaxel) combination, indicating that the apoptosis-inducing capacity of these drugs may not require either active P53 proteins or, possibly, induction of some of their targets (e.g. pro-apoptotic Bcl-2 family members). We are currently addressing these important questions.

\section{Conclusion}

Our results, based on a thorough approach that mobilized several independent technological platforms, demonstrated that the treatment of gastric cancer cells by lovastatin $+/-$ docetaxel triggered profound metabolomics and lipidomics alterations at the beginning of overt apoptosis. Although the sugar dependency and consumption by cancer cells has been amply reported, our data stressed the fact that some metabolomics modifications that accounted for altered glucose metabolism could also occur in response to lipid-restricting drugs like statins. We identified several cases of overlap between metabolomics/lipidomics and transcript levels, indicating that some of the biochemical changes could have been contributed by modifications in gene expression. It also appeared that the pro-apoptotic effects of the (lovastatin + docetaxel) combination were able to surpass the rise in energy supply that would otherwise be sufficient to fulfill the metabolic needs of cancer cells. We speculate that targeting energy sources may not necessarily be sufficient to kill cancer cells in the environment of a living organism. Rather, inhibiting anti-apoptotic proteins at the same time may be a more efficient option. It may also be suggested that some of the metabolites/lipids-and the genes that participated in producing them-that were modified, could provide novel biomarkers, or possibly pertinent targets, involved in the response to apoptosis-promoting treatments in a more general sense. 


\section{Material \& methods}

\section{Cell culture and treatments}

HGT-1 human gastric cancer cells were grown in 90 mm plates at $37^{\circ} \mathrm{C}$ under a humidified atmosphere of $5 \%$ $\mathrm{CO}_{2}$ in DMEM (Dulbecco's modified Eagle's medium) (Lonza, Saint Beauzire, France), containing $4.5 \mathrm{~g} \mathrm{~L}^{-1}$ glucose and supplemented with 5\% fetal bovine serum without antibiotics (Gibco-Invitrogen, Cergy-Pontoise, France $)^{12,36}$. AGS and HCT116 human gastric cancer and colon cancer cells, respectively, were grown under the same conditions in DMEM containing $4.5 \mathrm{~g} \mathrm{~L}^{-1}$ glucose and supplemented with $5 \%$ fetal bovine serum without antibiotics. The medium was changed at day +2 and day +4 . At day +4 , the culture medium was replaced by DMEM with L-glutamine, without phenol red, for the time of treatment, as it would otherwise saturate the signal, particularly in liquid chromatography-mass spectrometry (LC-MS). Cells were treated at $80 \%$ (roughly 10 million cells) cell confluence, with lovastatin $(12.5 \mu \mathrm{M}$, final concentration) and / or docetaxel ( $5 \mathrm{nM})$ for up to $36 \mathrm{~h}$. DMSO (Dimethyl sulfoxide), the vehicle of lovastatin, was added to all cell plates at the same concentration (less than $0.5 \% \mathrm{~V} / \mathrm{V}$ ) with no apparent toxicity observed in DMSO only-treated cells used as controls. At the morphological level, docetaxel-treated cells showed no alterations. By contrast, lovastatin or (docetaxel + lovastatin)-treated cells did show signs of initiating apoptosis (cell blebs) after $36 \mathrm{~h}$ whereas no such signs were observed after $24 \mathrm{~h}$. Adherent cells were rinsed twice with $10 \mathrm{~mL}$ cold phosphate buffered saline and $5 \mathrm{~mL}$ of icecold methanol were added per dish to stop any metabolic process and lyse the cells in situ. Cells were then centrifuged and the cell pellets were rinsed with $1 \mathrm{~mL}$ of icecold methanol. Pellets were subjected to sonication on ice $(2 \times 15 \mathrm{~s}$ at $70 \mathrm{~Hz})$ and distributed into aliquots. For protein assays, $0.1 \mathrm{~mL}$ was collected. All the platforms received $1.5 \mathrm{~mL}$ cell pellet fractions. Three independent biological replicates were prepared several weeks apart, and sent under the same conditions to all platforms.

\section{Determination of HMG-CoA reductase activity}

Cell pellets ( 4 million cells) were dissolved in $200 \mu \mathrm{l}$ buffer containing $50 \mathrm{mM} \mathrm{NaH}{ }_{2} \mathrm{PO}_{4}, 10 \mathrm{mM}$ EDTA, 0.1 $\mathrm{mM}$ DTT (pH 7.4). Leupeptin $(10 \mu \mathrm{g} / \mathrm{mL})$ and PMSF (1 $\mu \mathrm{L} / \mathrm{mL}$ of $1 \mathrm{M}$ PMSF in DMSO) were added just before use. Cells were disrupted by 20 passages in a glass/glass dounce followed by $20 \mathrm{~s}$ sonication. After a $15 \mathrm{~min}$ centrifugation at $1500 \times g$, the supernatants were removed and stored at $-80^{\circ} \mathrm{C}$ until use. Protein concentration was determined according to Bradford ${ }^{37}$.

HMG-CoA Red was measured essentially as described ${ }^{38}$ with some modifications. Incubation mixtures $(200 \mu \mathrm{L})$ contained buffer $\left(100 \mathrm{mM} \mathrm{KH} \mathrm{PO}_{4}, 50 \mathrm{mM} \mathrm{KCl}, 1 \mathrm{mM}\right.$ EDTA, and $5 \mathrm{mM}$ DTT, pH 7.4), $1.7 \mathrm{nmol}$ of $\left[{ }^{14} \mathrm{C}\right] \mathrm{HMG}-$
CoA and $8.3 \mathrm{nmol}$ of HMG-CoA (final concentration of $50 \mu \mathrm{M})$. The enzyme reactions were started by the addition of $200 \mu \mathrm{g}$ of proteins and $2.5 \mathrm{mM}$ NADPH. After 60 min incubation at $37^{\circ} \mathrm{C}$, reactions were terminated by adding $30 \mu \mathrm{L}$ of $72 \%$ trichloracetic acid. After $30 \mathrm{~min}$ to allow conversion of mevalonate to mevalonolactone, samples were centrifuged $3 \mathrm{~min}$ at $16000 \times g$ and $40 \mu \mathrm{L}$ of supernatant were used for HPLC analysis. In each sample, the HMG-CoA Red activity was determined in duplicate.

Samples $(30 \mu \mathrm{L})$ were injected and separated on a reverse phase Nucleosil C18 column $(5 \mu \mathrm{m}$ particle size, $250 \times 4.6 \mathrm{~mm}$ equipped with a $5 \mathrm{~mm}$ guard column of the same phase) with the following linear gradient of potassium phosphate $50 \mathrm{mM}$ pH6.8 (solvent A) and methanol (solvent B): $5 \%$ B for $2 \mathrm{~min}, 5-32 \% \mathrm{~B}$ up to $16 \mathrm{~min}, 32-5 \%$ for $10 \mathrm{~min}$, at a flow rate of $0.8 \mathrm{~mL} / \mathrm{min}$. The HPLC chromatograph was interfaced with a Flo-one Beta radiometric detector (Packard, Meriden, USA). Peak areas were calculated from the percentage of metabolite area to the total product area (metabolite + residual substrate). Data were expressed as $\mathrm{pmol} / \mathrm{min} / \mathrm{mg}$ of protein.

\section{Metabolomics and lipidomics analytical methods (see Supplementary information)}

Five instrumental platforms contributed to the analyses: NMRn ${ }^{\circ}$ (1H-NMR, Bruker), LC-MSn ${ }^{\circ} 1$ (UHPLC-LTQOrbitrap, Thermo), LC-MSn 2 (UHPLC-Exactive, Thermo), LC-MSn 3 (UPLC-HRMSe, Synapt Q-TOF G2, Waters), GC-MSn ${ }^{\circ} 1$ (GC-MS, Agilent). From extraction to analysis, each analytical platform processed the samples using its in-house procedure (Supplementary Table 3). Altogether, the analyses, i.e. metabolomics, lipidomics, in both positive or negative ionization modes (for MS), resulted in 9 data matrices from 5 platforms. Each platform generated its own dataset in a tabular format where variables were the peak area of a $\mathrm{m} / \mathrm{z}$ index for the mass spectrometry analysis or a bucket for NMR. These datasets included the 3 replicates for each treatment (control, lovastatin, docetaxel and co-treatment lovastatin + docetaxel), the analytical blank samples, and quality control samples (QC). QC samples were generated from an inhouse protocol in varying numbers depending on each platform (none for NMRn ${ }^{\circ} 1,10$ for LC-MSn ${ }^{\circ} 1, \mathrm{LC}-\mathrm{MSn}^{\circ} 2$ and $\mathrm{GC}-\mathrm{MSn}^{\circ} 1,9$ for LC-MSn $\left.{ }^{\circ} 3\right)$. They were prepared by making a mixture of all samples (controls and tests). They were used to correct the analytic drift, to filter the data and to evaluate statistics validity (reproducible profiles). They were introduced as follows: 5 at run start, then every 5-10 samples, depending on the separation sequence, and 3 at the end of the run (for GC). The relative standard deviation (RSD, \%) was calculated for QC variables peak areas to evaluate their analytical quality and robustness. Finally, lipid markers having a RSD value below 30\% in QC samples were kept for subsequent multivariate 
analysis. For the kinetics study, 34 selected biomarkers were quantified by GC-MS following the procedure described for the GC-MSn ${ }^{\circ} 1$ platform. Sphingomyelins and ceramides were quantified in samples by a validated assay on the LC-MSn 3 platform, as described previously $^{39}$. Additional platform protocols are presented in Supplementary Information.

\section{Data analysis}

Nine data matrices were generated by XCMS included in the workflow4metabolomics. We have collected all raw data from each platform, and we then have tuned each extraction parameter as finely as possible: one metabolomics data matrix in the positive electronic impact mode for $\mathrm{GC}-\mathrm{MSn}^{\circ} 1$, two metabolomic data matrices obtained in the positive and the negative ESI mode for $\mathrm{LC}-\mathrm{MSn}^{\circ} 1$, two metabolomic data matrices and two lipidomic data matrices generated in the positive and the negative ESI mode for $\mathrm{LC}-\mathrm{MSn}^{\circ} 2$, one lipidomic data matrix obtained in the positive ESI mode for LC-MSn ${ }^{\circ} 3$ and one metabolomic data matrix for $\mathrm{NMRn}^{\circ} 1$ platform. For each of the data matrices generated, a batch correction was applied to minimize the signal drift considering the inherent QCs.

Then, to compare these data matrices, a normalization step was introduced, i.e. combining all the generated data to be analyzed in the same order of magnitude. Mass spectrometry data were first normalized by a $\log _{10}$ transformation, then all datasets (including MS and NMR) were Pareto transformed ${ }^{13}$.

The relationship between the data tables from the different platforms were analyzed using the RV coefficient ${ }^{40,41}$. This RV coefficient can be interpreted as a multivariate equivalent of the coefficient of determination, which is a measure of the quality of the prediction of a linear regression $\left(R^{2}\right)$ ranging from [0-1]. When considering two matrices $\mathrm{X}$ and $\mathrm{Y}$, a coefficient $\mathrm{RV}$ equaling to 1 means that the relative position of the samples in $\mathrm{X}$ is similar to that in Y. In other words, the information contained in the two tables is identical. For each data matrix generated, a principal component analysis (PCA) was also performed with FactoMineR and the FactoExtra package working under the $\mathrm{R}$ computing environment ${ }^{42}$. The coordinates of the first dimension (Dim 1) of each PCA describing a data matrix were combined into a single file. We felt that multivariate tests would provide a fair description of the phenomena. Nevertheless, all univariate comparisons were also performed, which showed the same results, i.e. the equivalence of the control and docetaxel condition, the equivalence of the lovastatin and lovastatin + docetaxel condition, and the clear separation of the control and lovastatin, the control and lovastatin + docetaxel, the docetaxel and the lovastatin, the docetaxel and the lovastatin + docetaxel conditions. Univariate determinations are reported in Supplementary Tables 1 and 2. It could be observed that all platform determinations were quite homogeneous, i.e. obtained similar trends of variations for these metabolites. In addition, glutamine was the most strongly increased metabolite in response to the combination of lovastatin and docetaxel. However, some even stronger increases occurred for a few metabolites detected by a given platform, LCMS vs. GCMS, e.g. citric acid (10.22-fold according to LCMS, but 1.75 -fold according to GCMS) or glutathione (4.55fold according to LCMS and 2.1-fold according to GCMS). All statistical experiments were also performed with FactoMineR or Workflow4Metabolomics 3.0 (workflow4metabolomics.org).

\section{Acknowledgements}

This work is dedicated to our dear colleague Christophe Caron who has been instrumental in the development of the Galaxy pipeline for Metabolomics studies. This work was supported by Id2 Santé Bretagne, the INSERM, and the Université de Brest. We thank Biogenouest, particularly the Corsaire network of analytical platforms, for their support of this collaborative project. We also thank the support from the IDEALG project (P. Potin) and the Régions Bretagne and Pays de Loire.

\section{Author details}

'Génétique, Génomique Fonctionnelle et Biotechnologies, INSERM, Université de Brest, EFS, Brest, France. ${ }^{2}$ INRA UMR 1280, Centre de Recherche en Nutrition Humaine de l'Ouest (CRNHO), CHU Hôtel-Dieu, Université de Nantes, Nantes, France. ${ }^{3}$ CEISAM UMR 6230, EBSI Team, Université de Nantes, CNRS, Nantes, France. ${ }^{4}$ Laboratoire d'Etude des Résidus et Contaminants dans les aliments (LABERCA), Ecole Nationale Vétérinaire, Agroalimentaire et de l'Alimentation Nantes Atlantique (Oniris), Nantes, France. ${ }^{5}$ Institut de Génétique,

Environnement et Protection des Plantes, UMR 1349 INRA-Agrocampus OuestUniversité de Rennes 1, Rennes-Le Rheu, France. ${ }^{6}$ Team Biochemistry of Algal Defenses, UMR 7139 CNRS-UPMC \& LIA-DIAMS, Station Biologique, Roscoff,

France. ${ }^{7}$ Institut Universitaire de France, Paris, France

Conflict of interest

The authors declare that they have no conflict of interest.

\section{Publisher's note}

Springer Nature remains neutral with regard to jurisdictional claims in published maps and institutional affiliations.

Supplementary Information accompanies this paper at (https://doi.org/ 10.1038/s41419-018-0761-0).

Received: 21 December 2017 Revised: 18 May 2018 Accepted: 25 May 2018 Published online: 03 July 2018

\footnotetext{
References

1. Follet, J. et al. The association of statins and taxanes: an efficient combination trigger of cancer cell apoptosis. Br. J. Cancer 106, 685-692 (2012).

2. Pavlova, N. N. \& Thompson, C. B. The emerging hallmarks of cancer metabolism. Cell. Metab. 23, 27-47 (2016).

3. Annibaldi, A. \& Widmann, C. Glucose metabolism in cancer cells. Curr. Opin. Clin. Nutr. Metab. Care 13, 466-470 (2010).

4. Beloribi-Djefaflia, S., Vasseur, S. \& Guillaumond, F. Lipid metabolic reprogramming in cancer cells. Oncogenesis 5, e189 (2016).

5. Santos, C. R. \& Schulze, A. Lipid metabolism in cancer. FEBS J. 279, 2610-2623 (2012).

6. Hajar, R. Statins: past and present. Heart Views 12, 121-127 (2011).
} 
7. Matusewicz, L., Meissner, J., Toporkiewicz, M. \& Sikorski, A. F. The effect of statins on cancer cells-review. Tumour Biol. 36, 4889-4904 (2015).

8. Stryjkowska-Gora, A., Karczmarek-Borowska, B., Gora, T. \& Krawczak, K. Statins and cancers. Contemp. Oncol. (Pozn.) 19, 167-175 (2015).

9. Teramoto, $\mathrm{H}$. et al. Signaling from the small GTP-binding proteins Racl and Cdc42 to the c-Jun N-terminal kinase/stress-activated protein kinase pathway. A role for mixed lineage kinase 3/protein-tyrosine kinase 1, a novel member of the mixed lineage kinase family. J. Biol. Chem. 271, 27225-27228 (1996).

10. Cortes, J. E. \& Pazdur, R. Docetaxel. J. Clin. Oncol. 13, 2643-2655 (1995).

11. Ringel, I. \& Horwitz, S. B. Studies with RP 56976 (taxotere): a semisynthetic analogue of taxol. J. Natl. Cancer Inst. 83, 288-291 (1991).

12. Gibot, L. et al. Human caspase 7 is positively controlled by SREBP-1 and SREBP2. Biochem. J. 420, 473-483 (2009).

13. Martin, J. C. et al. Can we trust untargeted metabolomics? Results of the metabo-ring initiative, a large-scale, multi-instrument inter-laboratory study. Metabolomics 11, 807-821 (2015).

14. van der Kloet, F. M., Bobeldijk, I., Verheij, E. R. \& Jellema, R. H. Analytical error reduction using single point calibration for accurate and precise metabolomic phenotyping. J. Proteome Res. 8, 5132-5141 (2009).

15. Robert, $\mathrm{O}$. et al. pH optimization for a reliable quantification of brain tumor cell and tissue extracts with (1)H NMR: focus on choline-containing compounds and taurine. Anal. Bioanal. Chem. 399, 987-999 (2011).

16. Bligh, E. G. \& Dyer, W. J. A rapid method of total lipid extraction and purification. Can. J. Biochem. Physiol. 37, 911-917 (1959).

17. Kuhn, M. et al. STITCH 2: an interaction network database for small molecules and proteins. Nucleic Acids Res. 38(Database issue), D552-D556 (2010).

18. Shannon, P. et al. Cytoscape: a software environment for integrated models of biomolecular interaction networks. Genome Res. 13, 2498-2504 (2003).

19. Bindea, G. et al. ClueGO: a Cytoscape plug-in to decipher functionally grouped gene ontology and pathway annotation networks. Bioinformatics $\mathbf{2 5}$ 1091-1093 (2009).

20. Garcia-Alcalde, F., Garcia-Lopez, F., Dopazo, J. \& Conesa, A. Paintomics: a web based tool for the joint visualization of transcriptomics and metabolomics data. Bioinformatics 27, 137-139 (2011).

21. Alberts, A. W. Discovery, biochemistry and biology of lovastatin. Am. J. Cardiol. 62, 10J-15J (1988).

22. Henwood, J. M. \& Heel, R. C. Lovastatin. A preliminary review of its pharmacodynamic properties and therapeutic use in hyperlipidaemia. Drugs 36, 429-454 (1988)

23. Wang, R. W. et al. Biotransformation of lovastatin. IV. Identification of cytochrome P450 3A proteins as the major enzymes responsible for the oxidative metabolism of lovastatin in rat and human liver microsomes. Arch. Biochem. Biophys. 290, 355-361 (1991).

24. Baker, S. D. Drug interactions with the taxanes. Pharmacotherapy 17(5 Pt 2), 126S-132S (1997).
25. Zhou, Q., Luo, A. \& Kummerow, F. A. Lovastatin reversed the enhanced sphingomyelin caused by 27-hydroxycholesterol in cultured vascular endothelial cells. Biochem. Biophys. Rep. 5, 127-133 (2016).

26. Haimovitz-Friedman, A., Kolesnick, R. N. \& Fuks, Z. Ceramide signaling in apoptosis. Br. Med. Bull. 53, 539-553 (1997).

27. Henry, B., Moller, C., Dimanche-Boitrel, M. T., Gulbins, E. \& Becker, K. A. Targeting the ceramide system in cancer. Cancer Lett. 332, 286-294 (2013).

28. Huang, C. \& Freter, C. Lipid metabolism, apoptosis and cancer therapy. Int. J. Mol. Sci. 16, 924-949 (2015).

29. Modrak, D. E., Leon, E., Goldenberg, D. M. \& Gold, D. V. Ceramide regulates gemcitabine-induced senescence and apoptosis in human pancreatic cancer cell lines. Mol. Cancer Res. 7, 890-896 (2009).

30. Binnington, B. et al. Inhibition of Rab prenylation by statins induces cellular glycosphingolipid remodeling. Glycobiology 26, 166-180 (2016).

31. Vaishampayan, U., Parchment, R. E., Jasti, B. R. \& Hussain, M. Taxanes: an overview of the pharmacokinetics and pharmacodynamics. Urology $54(6 \mathrm{~A}$ Suppl), 22-29 (1999).

32. Ganansia-Leymarie, V., Bischoff, P., Bergerat, J. P. \& Holl, V. Signal transduction pathways of taxanes-induced apoptosis. Curr. Med. Chem. Anticancer Agents $\mathbf{3}$, 291-306 (2003)

33. L'Esperance, S., Bachvarova, M., Tetu, B., Mes-Masson, A. M. \& Bachvarov, D. Global gene expression analysis of early response to chemotherapy treatment in ovarian cancer spheroids. BMC Genomics 9, 99 (2008).

34. Kobayashi, Y. et al. Drug repositioning of mevalonate pathway inhibitors as antitumor agents for ovarian cancer. Oncotarget 8, 72147-72156 (2017).

35. Zhang, J. \& Pavlova, N. N. \& Thompson, C. B. Cancer cell metabolism: the essential role of the nonessential amino acid, glutamine. EMBO J. 36, 1302-1315 (2017).

36. Laboisse, C. L. et al. Characterization of a newly established human gastric cancer cell line HGT-1 bearing histamine H2-receptors. Cancer Res. 42 1541-1548 (1982).

37. Bradford, M. M. A rapid and sensitive method for the quantitation of microgram quantities of protein utilizing the principle of protein-dye binding. Anal. Biochem. 72, 248-254 (1976)

38. Houten, S. M., Schneiders, M. S., Wanders, R. J. \& Waterham, H. R. Regulation of isoprenoid/cholesterol biosynthesis in cells from mevalonate kinase-deficient patients. J. Biol. Chem. 278, 5736-5743 (2003).

39. Croyal, M. et al. Fenofibrate decreases plasma ceramide in type 2 diabetes patients: a novel marker of CVD?. Diabetes Metab. 44, 143-149 (2018).

40. Robert, P. \& Escouffier, Y. A unifying tool for linear multivariate statistical methods: the RV-coefficient. Appl. Stat. 25, 257-265 (1976).

41. Lavit, C., Escouffier, Y., Sabatier, R. \& Traissac, P. The ACT (STATIS method) Comput. Stat. Data Anal. 18, 97-119 (1994).

42. Lê, S., Josse, P. \& Husson, F. FactoMineR: an R package for multivariate analysis. J. Stat. Softw. 25, 1-18 (2008). 